\title{
Prostaglandin Endoperoxides Modulate the Response to Thromboxane Synthase Inhibition during Coronary Thrombosis
}

Desmond J. Fitzgerald, James Fragetta, and Garret A. FitzGerald

Divisions of Clinical Pharmacology and Cardiology, Vanderbilt University, Nashville, Tennessee 37232

\begin{abstract}
Prostaglandin endoperoxides $\left(\mathrm{PGG}_{2} / \mathrm{PGH}_{2}\right)$, precursors of thromboxane (TX) $A_{2}$ and prostaglandins, may accumulate sufficiently in the presence of a TXA synthase inhibitor to exert biological activity. To address whether this modulates the response to $T X A_{2}$ synthase inhibition in the setting of thrombosis in vivo, we examined the interaction of a $T X \mathbf{A}_{2}$ synthase inhibitor (U63,557a) and a TXA $/$ prostaglandin endoperoxide receptor antagonist $(\mathbf{L 6 3 6 , 4 9 9 )}$ in a canine model of coronary thrombosis after electrically induced endothelial injury. U63,557a exerted little inhibitory effect in this model despite a marked reduction in serum $\mathbf{T X B}_{2}$ and urinary 2,3dinor-TXB $\mathbf{T H}_{2}$, an index of $\mathrm{TXA}_{2}$ biosynthesis. Combination of the two drugs was more effective than either drug alone. The enhanced effect achieved upon addition of the TX $\mathbf{A}_{2} /$ prostaglandin endoperoxide receptor antagonist to the $T X \mathbf{A}_{2}$ synthase inhibitor suggests that the response to the latter compound was limited by the proaggregatory effects of prostaglandin endoperoxides. The increased effect of the combination over the receptor antagonist alone may reflect metabolism of $\mathbf{P G G}_{2} / \mathbf{P G H}_{2}$ to platelet inhibitory prostaglandins. This is supported by the following findings: (a) urinary 2,3-dinor-6keto-PGF , $_{1 \alpha}$, index of prostacyclin biosynthesis, increased after administration of the synthase inhibitor, an effect that was exaggerated in the presence of thrombosis; $(b)$ inhibition of arachidonate-induced platelet aggregation by U63,557a was dependent on the formation of a platelet-inhibitory prostaglandin; and $(c)$ pretreatment with aspirin abolished the synergism between these compounds. These studies demonstrate that prostaglandin endoperoxides modulate the response to $T X \mathbf{A}_{2}$ synthase inhibition in vivo and identify a drug combination of potential therapeutic efficacy in the prevention of thrombosis.
\end{abstract}

\section{Introduction}

Thromboxane (TX) $A_{2}$, the major cyclooxygenase product of arachidonic acid in platelets, is a potent platelet aggregant and vasoconstrictor (1) and plays a pathogenic role in a variety of ischemic heart disease syndromes (2-6). A reduction in $\mathrm{TXA}_{2}$ biosynthesis may be achieved by inhibition of $\mathrm{TXA}_{2}$ synthase, the enzyme that catalyses the conversion of prostaglandin endoperoxides $\left(\mathrm{PGG}_{2} / \mathrm{PGH}_{2}\right)$ to $\mathrm{TXA}$ (7-9). This has a theoretical advantage over cyclooxygenase inhibition, most com-

Address reprint requests to Dr. D. J. Fitzgerald, Division of Clinical Pharmacology, Vanderbilt University, Nashville, TN 37232. $R$
1988

Received for publication 5 April 1988 and in revised form 27 June

J. Clin. Invest.

(c) The American Society for Clinical Investigation, Inc.

0021-9738/88/11/1708/06 \$2.00

Volume 82, November 1988, 1708-1713 monly achieved with aspirin, in that it preserves prostacyclin $\left(\mathrm{PGI}_{2}\right)$ biosynthesis (7-9). $\mathrm{PGI}_{2}$ is a major cyclooxygenase product of arachidonic acid in vascular endothelium and is a potent platelet inhibitor and vasodilator (10). Evidence from experimental studies suggests that $\mathrm{PGI}_{2}$ limits platelet activation in vivo $(11,12)$. Furthermore, $\mathbf{P G I}_{2}$ biosynthesis is increased in conditions associated with platelet activation, including severe atherosclerosis (13), unstable angina (2), and systemic sclerosis (14). Coincident inhibition of $\mathrm{PGI}_{2}$ formation, therefore, may limit the therapeutic response to aspirin and other cyclooxygenase inhibitors. In contrast, $\mathrm{TXA}_{2}$ synthase inhibitors do not inhibit $\mathrm{PGI}_{2}$ biosynthesis (7-9).

Despite their biochemical selectivity, $\mathrm{TXA}_{2}$ synthase inhibitors are weak platelet inhibitors in vitro $(7,15-17)$ and exert variable effects in vivo (18-21). This may reflect the continued formation of platelet prostaglandin endoperoxides that activate a receptor, shared with $\mathrm{TXA}_{2}$, mediating platelet activation and vasoconstriction $(7,15,17)$. Indeed, the antiplatelet effects of TXA $_{2}$ synthase inhibitors may be largely mediated through platelet-inhibitory prostaglandins formed by the metabolism of accumulated prostaglandin endoperoxides. Marcus et al., and Schafer et al., have demonstrated that, in the presence of a TXA 2 synthase inhibitor, platelet-derived prostaglandin endoperoxides may be metabolized by contiguous endothelium to $\mathrm{PGI}_{2}(22,23)$. Other products, including $\mathrm{PGE}_{2}$ and $\mathrm{PGD}_{2}$, may also be formed and mediate the platelet effects of TXA $_{2}$ synthase inhibitors $(24,25)$. Pharmacologic studies have also implied a role for vascular-derived prostaglandins in mediating the response to $\mathrm{TXA}_{2}$ synthase inhibition in vivo $(11,26)$. Thus, prostaglandin endoperoxides may modulate the response to $\mathrm{TXA}_{2}$ synthase inhibitors, both by substituting for the proaggregatory effects of $\mathrm{TXA}_{2}$ and through their metabolism to platelet-inhibitory prostaglandins.

To determine if these mechanisms influence thrombogenesis in vivo, we have examined the interaction of a $\mathrm{TXA}_{2}$ synthase inhibitor and an antagonist of the shared $\mathrm{TXA}_{2} /$ prostaglandin endoperoxide receptor in a canine model of coronary artery thrombosis $(27,28)$. These studies demonstrate biochemical and functional evidence of a role for prostaglandin endoperoxides in modulating the response to $\mathrm{TXA}_{2}$ synthase inhibitors in vivo and identify an interaction of potential therapeutic importance between these classes of drugs.

\section{Methods}

\section{Animal studies}

Acute experiments. All animal studies were reviewed and approved by the Animal Care Committee at Vanderbilt University. Male mongrel dogs (17-23 kg) were anesthetized with pentobarbitone $30 \mathrm{mg} / \mathrm{kg}$ and ventilated using a Harvard respirator (Harvard Apparatus Co., Natick, MA). The circumflex coronary artery was isolated through a left thoracotomy and all branches ligated down to the first obtuse marginal branch. A needle electrode was passed through the arterial wall such that $4-5 \mathrm{~mm}$ of exposed wire lay against the endothelium, as pre- 
viously described $(27,28)$. The electrode consisted of 30-gauge Teflon coated silver wire onto the end of which was crimped the tip $(2 \mathrm{~mm})$ of a 28-gauge hypodermic needle to aid passage through the vessel wall. The electrode was connected in series with a $50,000 \Omega$ potentiometer, a $20,000 \Omega$ resistor, an ammeter and the positive terminal of a 9-V battery, and the circuit was grounded to the subcutaneous tissues of the dog. An electromagnetic flow probe (Carolina Instruments, King, NC) was positioned proximal to the electrode site and connected to an electromagnetic flowmeter (SP 2202; Gould-Statham Instrument Co., Inc., Hato Rey, Puerto Rico). Blood pressure was recorded through a polyethylene catheter implanted in the femoral artery. Coronary blood flow and femoral artery pressure were recorded continuously throughout the experiment using a strip chart recorder (HP 1758A; Hewlett Packard Co., Waltham, MA).

Animals were randomized to one of four treatments. Group I animals received vehicle only and acted as controls. In group II, the TXA synthase inhibitor, 5-(3'-pyridinylmethyl) benzfuran-2-carboxylate (U63,557a), was administered in a bolus dose of $10 \mathrm{mg} / \mathrm{kg}$ i.v. in normal saline over $10 \mathrm{~min}$. In group III, the $\mathrm{TXA}_{2} /$ prostaglandin endoperoxide receptor antagonist, 3-carboxyl-dibenzo (b,f) thiepin-5,5dioxide $(L 636,499)$, was administered in a bolus dose of $20 \mathrm{mg} / \mathrm{kg}$ followed by $2 \mathrm{mg} / \mathrm{kg}$ per min. In group IV, both U63,557a and L636,499 were administered in these doses.

After a stabilization period of $30 \mathrm{~min}$, drug or vehicle was administered. After a further $\mathbf{3 0}$ min coronary thrombosis was induced by passing a 150-200 $\mu \mathrm{A}$ current through the electrode and the animal observed for $4 \mathrm{~h}$ or until complete coronary occlusion had occurred. The time to coronary occlusion, defined as the time from the onset of current to the point at which zero flow was achieved, was compared in the four groups. Blood was obtained before and hourly following drug administration for platelet aggregation studies and for measurement of serum $\mathrm{TXB}_{2}$.

Chronic experiments. In chronic studies, the same surgical procedure was used with the exception that an ultrasonic flow probe (Biomedical Engineering, Iowa University, Iowa City, IA) was implanted in place of the electromagnetic flow probe. The terminals of the flow probe and the electrode were brought to the surface in a subcutaneous pouch, the chest closed and the animal allowed to recover. Heparin (100 IU/kg s.c.) was administered every $8 \mathrm{~h}$ for $48 \mathrm{~h} .5 \mathrm{~d}$ after surgery, the animal was sedated with acepromazine $1 \mathrm{mg} / \mathrm{kg}$ and morphine sulfate $1-2 \mathrm{mg} / \mathrm{kg}$. The electrode terminal was recovered and connected to the circuit described above. The terminals of the ultrasonic flow probe were connected to a directional pulsed Doppler flowmeter (545C-4, Biomedical Engineering, Iowa University). Arterial blood pressure was not recorded in these experiments to avoid traumatic increases in eicosanoid biosynthesis.

Animals were randomized to the same study groups as in the acute experiments. An additional group (group $\mathrm{V}$ ) of animals received aspirin $20 \mathrm{mg} / \mathrm{kg}$ in $0.1 \mathrm{M} \mathrm{Na}_{2} \mathrm{CO}_{3}$ i.v. over $10 \mathrm{~min}$ before the administration of the combination of U63,557a and L636,499. Coronary thrombosis was induced by passing a $200 \mu \mathrm{A}$ current through the electrode and the animal observed for a maximum of $7 \mathrm{~h}$ or until $2 \mathrm{~h}$ after complete coronary occlusion. Urine was collected by catheterization before drug administration and hourly thereafter for measurement of 2,3-dinor- $\mathrm{TXB}_{2}$ and 2,3-dinor-6-keto-prostaglandin (PG) $\mathrm{F}_{1 \alpha}$, major urinary metabolites of $\mathrm{TXA}_{2}$ and $\mathrm{PGI}_{2}$, respectively $(29,30)$.

\section{Platelet aggregation studies}

Platelet aggregation to arachidonic acid (0.075-0.66 mM) (Sigma Chemical Co., St. Louis, MO) and to the endoperoxide analogue, (15S)-hydroxy 11,9-(epoxymethano)-prostadienoic acid (U46619, 0.13-2.0 $\mu \mathrm{M}$ ) (Upjohn Co., Kalamazoo, MI) was determined in platelet-rich plasma (PRP) by light transmission (31), using a multichannel aggregometer (Biodata PAP-4, Biodata, Hartboro, PA). PRP was prepared by centrifuging citrated venous blood $(3.8 \% \mathrm{Na}$ citrate, $9: 1 \mathrm{vol} /$ vol) at $3,000 \mathrm{rpm}$ for $50 \mathrm{~s}$ and adjusted to 300,000 platelets/ $\mu 1$ with platelet-poor plasma obtained by centrifuging the remaining blood at $3,000 \mathrm{rpm}$ for $10 \mathrm{~min}$. Platelet aggregations were performed in $500-\mu \mathrm{l}$ aliquots of plasma with aggregating agents added in volumes of $10 \%$ or less. The concentration of aggregating agent is expressed as the final concentration in PRP. As canine platelets may fail to aggregate directly to arachidonic acid and U46619 in $30-50 \%$ of cases, the platelets were first primed with ADP at a concentration (1-2 $\mu \mathrm{M})$ that induced a small reversible wave of aggregation, as previously described (32).

\section{Biochemical studies}

Urinary 2,3-dinor $\mathrm{TXB}_{2}$ and 2,3-dinor-6-keto $\mathrm{PGF}_{1 \alpha}$ were determined by gas chromatography, negative ion-chemical ionization, mass spectrometry using their respective tetradeuterated analogues as internal standards $(30,33)$. Briefly, to a $5-\mathrm{ml}$ sample of urine was added $5 \mathrm{ng}$ of each internal standard and the urine extracted by immunoaffinity chromatography as previously described (34). After formation of the methoxime derivatives, further purification was achieved by thin-layer chromatography and the sample finally derivatized to the trimethylsilyl ether. Final separation and quantitation was achieved by a gas chromatograph in series with a Nermag R 10-10 operated in the negative ion mode.

Serum $\mathrm{TXB}_{2}$ was determined by radioimmunoassay following incubation of whole blood in a glass test tube at $37^{\circ} \mathrm{C}$ for $45 \mathrm{~min}(35)$.

\section{Statistical analysis}

The time to coronary occlusion was compared in the different treatment groups by the Kruskal-Wallis one-way analysis of variance (36). This is a nonparametric test and makes no assumptions as to the distribution of the data. Animals who failed to occlude were assigned a value equal to the maximum period of observation. However, this does not alter the statistical analysis that is based on ranks and not absolute values. The effects of treatments on serum $\mathrm{TXB}_{2}$ and platelet aggregation were compared within groups by paired $t$ test and between groups by one-way analysis of variance. Urinary metabolites were analyzed by two-way analysis of variance within groups. All data are expressed as the mean \pm SEM.

\section{Results}

Serum $T X B_{2}$. U63,557a decreased serum $\mathrm{TXB}_{2}$ by $86 \pm 3.9 \%$ ( $n=9, P<0.001)$ when given alone and by $93 \pm 1.7 \%(n=7, P$ $<0.001$ ) in combination with L636,499. The effects of the two treatments were not significantly different from each other. This reduction in serum $\mathrm{TXB}_{2}$ persisted for at least $4 \mathrm{~h}$ in both groups. Pretreatment with aspirin exerted little further effect $(96 \pm 1 \%, n=6)$. In contrast, L636,499 induced only a small reduction in serum $\mathrm{TXB}_{2}(23 \pm 8 \%, n=14 ; P<0.05)$, which by analysis of variance was not significantly different from vehicle alone $(9 \pm 8 \%, n=8)$.

Animal studies. In acute, open-chest experiments heart rate and blood pressure were unaltered by U63,577a and L636,499 given individually or combined. Coronary occlusion occurred in control animals in $74 \pm 6.7 \mathrm{~min}$, similar to what we have reported previously (28). The time to coronary occlusion was prolonged $32 \pm 15 \%$ by U63,557a (Fig. 1) to $98 \pm 4.0 \mathrm{~min}$ ( $n$ $=17, \mathrm{NS})$. L636,499 had a more marked effect, prolonging the time to occlusion to $159 \pm 16 \min (n=12, P<0.01)$. Indeed, three of the dogs failed to occlude over the 4-h observation period. In dogs treated with the combination of the two drugs coronary occlusion was prevented in five of nine experiments, the time to coronary occlusion being prolonged by $204 \pm 8 \%$ to $225 \pm 6.2 \mathrm{~min}(P<0.001)$. Thus, combination of U63,557a and L636,499 was more effective in preventing and delaying coronary occlusion than either treatment alone $(P<0.05)$.

To further evaluate the effect of the combination therapy, we performed the same experiment in closed-chest animals where more prolonged observation was possible. Coronary oc- 


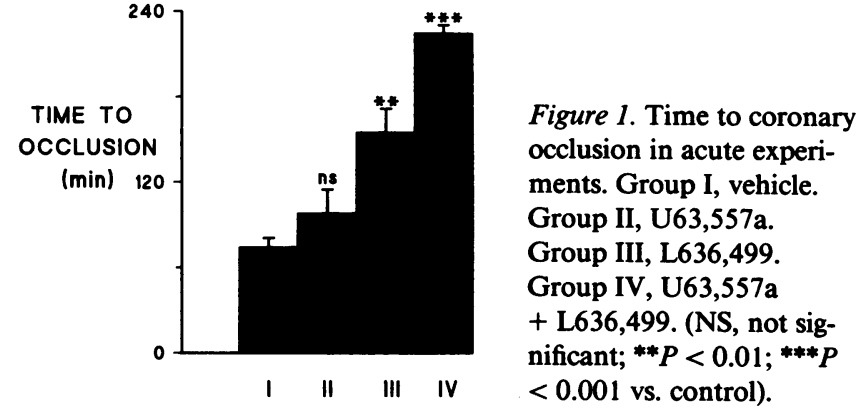

clusion occurred in $72 \pm 16 \mathrm{~min}(n=8)$ in vehicle-treated animals, similar to acute experiments. As in the acute study, the combination of U63,557a and L636,499 was more effective than either treatment alone (Fig. 2), five of nine animals failing to occlude over $7 \mathrm{~h}$ of observation with a mean time to coronary occlusion of $341 \pm 39 \mathrm{~min}(P<0.001$ vs. controls, $P$ $<0.01$ vs. either treatment). Thus, U63,557a increased the time to coronary occlusion to a greater extent in the presence of the $\mathrm{TXA}_{2}$ /prostaglandin endoperoxide receptor antagonist, L636,499 ( $181 \pm 21 \%$ vs. $71 \pm 30 \%, P<0.01)$, suggesting a synergistic interaction between these drugs. In contrast, the combination of U63,557a and L636,499 failed to alter the time to coronary occlusion in animals pretreated with aspirin $(69 \pm 6$ min, $n=5$ ).

Platelet studies. Platelet aggregation to the $\mathrm{TXA}_{2} /$ prostaglandin endoperoxide analogue, U46619, and to arachidonic acid was unaltered in control experiments (Figs. 3 and 4). L636,499 inhibited the response to U46619 and arachidonic acid, increasing the threshold concentration of arachidonic acid for complete, irreversible aggregation from $0.23 \pm 0.02$ to $0.77 \pm 0.09 \mathrm{mM}(n=16, P<0.001)$. Combination of U63,557a and L636,499 exerted a more marked effect, completely abolishing the response to both $\mathrm{U} 46619$ and arachidonic acid.

In contrast, U63,557a alone had no effect on U46619-induced platelet aggregation. The effect of U63,557a on arachidonate-induced platelet aggregation was dependent on the concentration of the agonist. Thus, inhibition of platelet aggregation was paradoxically greater at higher concentrations of arachidonic acid with no inhibition demonstrated at 0.08 and $0.17 \mathrm{mM}$ arachidonic acid (Fig. 4). This effect also occurred in vitro and was demonstrable with a structurally distinct $\mathrm{TXA}_{2}$ synthase inhibitor, imidazo [1,5-2] pyridine-5-hexanoic acid (CGS13080) (Fig. 5). Furthermore, platelets failed to aggregate to ADP or U46619 after incubation of PRP with U63,557a 10 $\mu \mathrm{g} / \mathrm{ml}$ and arachidonic acid $0.66 \mathrm{mM}$. This inhibition of plate-

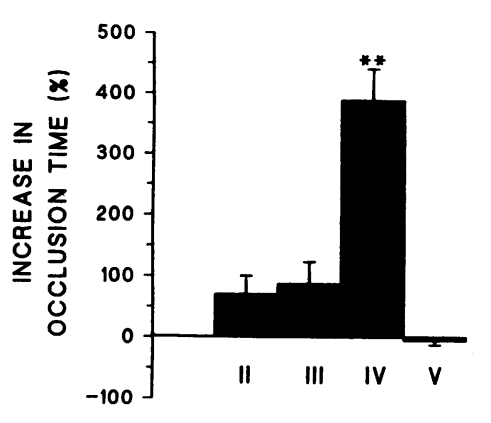

Figure 2. Percent increase in the time to coronary occlusion compared with vehicle-treated controls in chronic experiments. Group II, U63,557a. Group III, L636,499. Group IV, U63,557a + L636,499. Group V, aspirin + U63,557a + L636,499. $\left({ }^{* *} P<0.01\right.$ vs. groups II, III, and V).
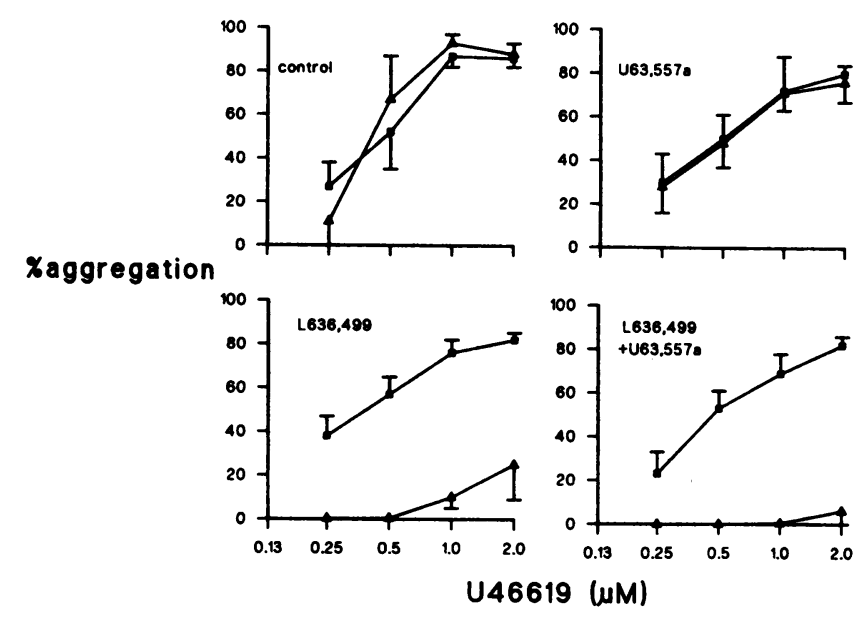

Figure 3. Ex vivo platelet aggregation to U46619. a, predrug. A, postdrug.

let aggregation was prevented by pretreating the platelets with aspirin $10 \mu \mathrm{M}$ for $10 \mathrm{~min}$, suggesting that this reflected formation of a cyclooxygenase product.

Biochemical studies. Urinary excretion of 2,3-dinor- $\mathrm{TXB}_{2}$ and 2,3-dinor-6-keto-PGF Pas $_{1 \alpha}$ wastermined only in chronic experiments where artifactual production of the parent eicosanoids by surgical trauma is minimized (37). In all groups, urinary metabolite excretion was not significantly different on the day of study from presurgery levels. Urinary 2,3-dinor$\mathrm{TXB}_{2}$ increased significantly during induction of coronary thrombosis, from $871 \pm 118$ to $1650 \pm 281 \mathrm{pg} / \mathrm{mg}$ creatinine $(P$ $<0.01)$ and remained elevated after complete coronary occlusion (Fig. 6). U63,557a prevented the increase in 2,3-dinor$\mathrm{TXB}_{2}$ excretion when given alone and when combined with L636,499. This persisted over the period of observation, up to $7 \mathrm{~h}$ in some experiments. Similarly, pretreatment with aspirin prevented the increase in excretion of this metabolite. In contrast, 2,3-dinor-TXB ${ }_{2}$ increased in dogs treated with L636,499 and continued to rise over the prolonged period of observation in these animals (Fig. 6).

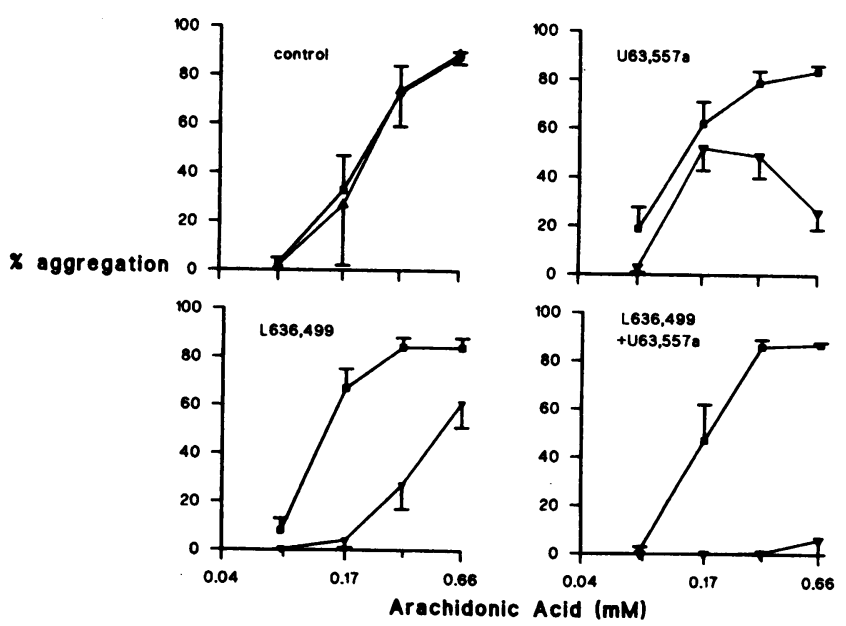

Figure 4. Ex vivo platelet aggregation response to arachidonic acid. n, predrug, $\Delta$, postdrug. 


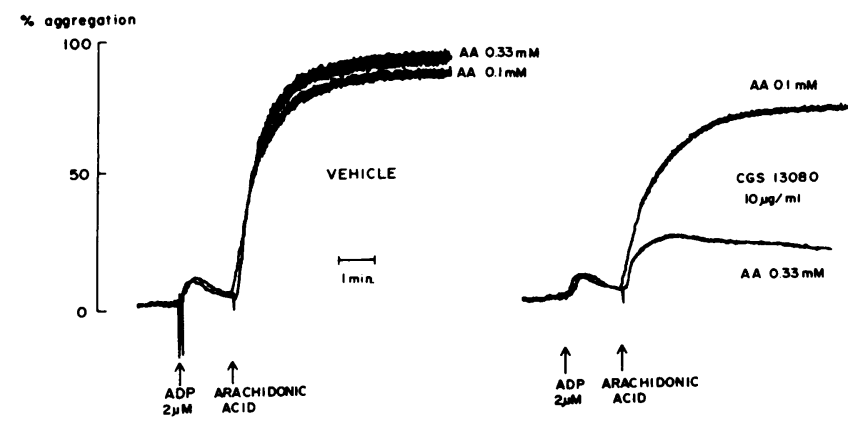

Figure 5. Platelet aggregation to arachidonic acid in canine PRP in the presence and absence of CGS13080 $10 \mu \mathrm{g} / \mathrm{ml}$. After incubation with the thromboxane synthase inhibitor platelet aggregation to 0.1 $\mathrm{mM}$ arachidonic acid is slightly inhibited. Increasing the concentration of arachidonic acid results in a more marked degree of platelet inhibition.

Urinary 2,3-dinor-6-keto-PGF ${ }_{1 \alpha}$ also increased both in control animals and in animals treated with L636,499 (Fig. 7), reaching a peak after complete coronary occlusion. This increase was more marked in dogs treated with U63,557a, particularly when combined with the $\mathrm{TXA}_{2}$ /prostaglandin endoperoxide receptor antagonist, L636,499, and persisted throughout the period of observation. Pretreatment with aspirin markedly blunted the increase in urinary 2,3-dinor-6-keto-PGF la $_{\alpha}$ seen with the combination therapy.

\section{Discussion}

These studies address the role of prostaglandin endoperoxides in modulating the response to $\mathrm{TXA}_{2}$ synthase inhibitors in a chronic canine model of coronary thrombosis. This is a $\mathrm{TXA}_{2}$ dependent model in that it is associated with increased thromboxane biosynthesis (37) and is inhibited by specific $\mathrm{TXA}_{2} /$ prostaglandin endoperoxide receptor antagonists (28). Biosynthesis of $\mathrm{TXA}_{2}$ and $\mathrm{PGI}_{2}$ was quantitated by excretion of their enzymatic metabolites in urine using highly specific assays. These methods avoid the artifacts inherent in the measurement of nonenzymatic, hydrolysis products $\left(\mathrm{TXB}_{2}\right.$ and 6-

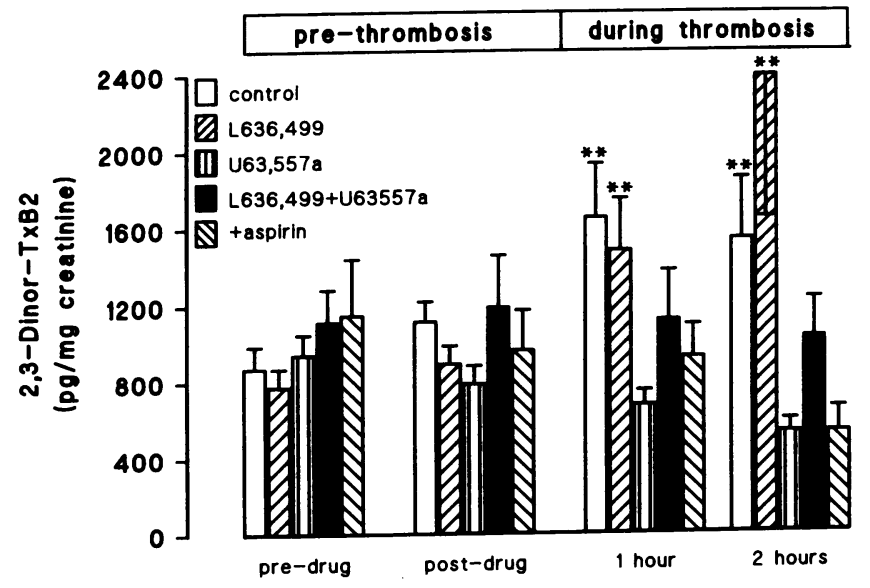

Figure 6. Urinary 2,3-dinor- $\mathrm{TXB}_{2}$ before and after drug administration and during induction of coronary thrombosis in the chronic canine model. ( ${ }^{* *} P<0.01$ vs. level before induction of coronary thrombosis.)

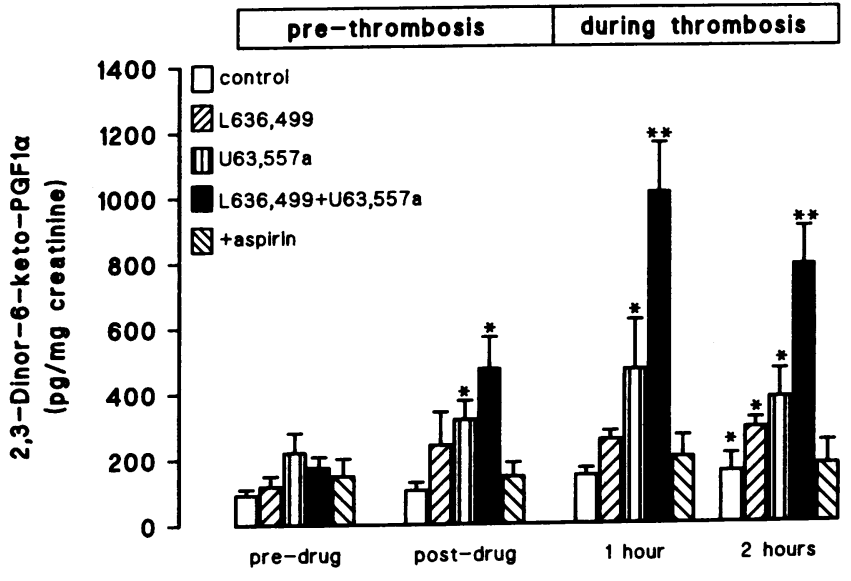

Figure 7. Urinary 2,3-dinor-6-keto-PGF ${ }_{1 \alpha}$ before and after drug administration and during induction of coronary thrombosis in the chronic canine model. $\left({ }^{*} P<0.05 ;{ }^{* *} P<0.01\right.$ vs. level before induction of coronary thrombosis).

keto-PGF ${ }_{1 \alpha}$, respectively) and the trauma of blood sampling (38). Furthermore, eicosanoid biosynthesis was determined remote from the trauma of surgery, when $\mathrm{TXA}_{2}$ and $\mathrm{PGI}_{2}$ formation had returned to baseline. In open-chest experiments, excretion of the enzymatic metabolites of $\mathrm{TXA}_{2}$ and $\mathrm{PGI}_{2}$ is markedly increased, reflecting an increase in the formation of the parent eicosanoids by traumatized tissue and platelet activation at wound sites (37). However, by $5 \mathrm{~d}$ postsurgery, eicosanoid biosynthesis had fallen to preoperative levels, allowing detection of a thrombus-related increase in $\mathrm{TXA}_{2}$ and $\mathrm{PGI}_{2}$ biosynthesis that would have been largely obscured in open-chest experiments.

To examine the role of prostaglandin endoperoxides in the response to $\mathrm{TXA}_{2}$ synthase inhibition in this model, we examined the interaction of $\mathrm{L} 636,499$ and U63,557a. L636,499 is a selective antagonist of the shared $\mathrm{TXA}_{2}$ /prostaglandin endoperoxide receptor $(39,40)$. Consistent with this, L636,499 did not inhibit $\mathrm{TXA}_{2}$ or $\mathrm{PGI}_{2}$ biosynthesis during coronary thrombosis in this model and prevented platelet aggregation to the endoperoxide analogue, U46619. The small reduction in serum $\mathrm{TXB}_{2}$ following the administration of L636,499 has been noted previously for this and other TXA $\mathrm{A}_{2}$ antagonists (28, $41,42)$ and may reflect inhibition of $\mathrm{TXA}_{2}$-dependent platelet activation during incubation of the blood. U63,557a is a potent $\mathrm{TXA}_{2}$ synthase inhibitor (43) and, in contrast to L636,499, markedly depressed serum $\mathrm{TXB}_{2}$, prevented the increase in $\mathrm{TXA}_{2}$ biosynthesis during coronary thrombosis and had no effect on U46619-induced platelet aggregation.

Despite their potent effects on thromboxane biosynthesis, $\mathrm{TXA}_{2}$ synthase inhibitors exhibit limited platelet inhibitory activity both in vitro and ex vivo $(7,15-20)$. Similarly, in this study, U63,557a failed to inhibit coronary occlusion despite its effects on $\mathrm{TXA}_{2}$ biosynthesis. Similar results have been reported in this model using a structurally distinct $\mathrm{TXA}_{2}$ synthase inhibitor (19). Addition of the $\mathrm{TXA}_{2} /$ prostaglandin endoperoxide receptor antagonist, L636,499, enhanced the re-

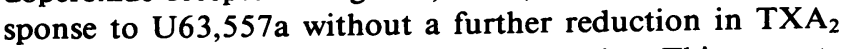
biosynthesis in vivo or in serum $\mathrm{TXB}_{2}$ ex vivo. This suggests that the response to the $\mathrm{TXA}_{2}$ synthase inhibitor was limited 
by either incomplete inhibition of $\mathrm{TXA}_{2}$ formation or by the accumulation of prostaglandin endoperoxides. Prostaglandin endoperoxides, precursors of $\mathrm{TXA}_{2}$ in the cyclooxygenase pathway of arachidonic acid metabolism, activate a receptor shared with $\mathrm{TXA}_{2}$ that induces platelet activation and vascular smooth muscle contraction $(15,44)$. In vitro studies demonstrate that prostaglandin endoperoxides can substitute for the proaggregatory effect of $\mathrm{TXA}_{2}$ and overcome the effect of TXA $_{2}$ synthase inhibition on platelets $(7,15-17)$. In view of the marked inhibition of $\mathrm{TXA}_{2}$ biosynthesis at doses of $\mathrm{U} 63,557 \mathrm{a}$ used in this study, these data are consistent with the hypothesis that prostaglandin endoperoxides also limit the response to $\mathrm{TXA}_{2}$ synthase inhibition in vivo.

This study also demonstrated that addition of the $\mathrm{TXA}_{2}$ synthase inhibitor increased the response to the $\mathrm{TXA}_{2} /$ prostaglandin endoperoxide receptor antagonist. In part this may reflect a reduction in agonist formation. Alternatively, it may be due to increased biosynthesis of platelet inhibitory prostaglandins. In vitro studies have demonstrated that prostaglandin endoperoxides derived from platelets in the presence of a TXA $_{2}$ synthase inhibitor may be metabolized or shunted to platelet-inhibitory prostaglandins, including $\mathrm{PGI}_{2}(22,23)$ and $\mathrm{PGD}_{2}(24,25)$, by contiguous tissues and by the isomerase activity of plasma albumin, respectively. Indeed, this may be a major mechanism of their activity both in vitro (25) and in vivo (11). As evidence of a shunt to platelet inhibitory prostaglandins, there was a marked increase in $\mathrm{PGI}_{2}$ formation, coincident with the inhibition of $\mathrm{TXA}_{2}$ biosynthesis in animals treated with U63,557a alone or in combination with the $\mathrm{TXA}_{2} /$ prostaglandin endoperoxide receptor antagonist. This effect was most marked during induction of coronary thrombosis. Furthermore, the enhanced effect seen when U63,557a was combined with L636,499 was abolished by aspirin at a dose that prevented the increase in $\mathrm{PGI}_{2}$ biosynthesis. These data, therefore, provide both functional and biochemical evidence of a shunt to platelet-inhibitory prostaglandins in the presence of a $\mathrm{TXA}_{2}$ synthase inhibitor. Similar findings have recently been reported in human volunteers by Gresele and co-workers (26). In their studies, addition of a $\mathrm{TXA}_{2}$ synthase inhibitor enhanced the effect of a $\mathbf{T X A}_{2}$ /prostaglandin endoperoxide receptor antagonist on bleeding time, an effect that was prevented by pretreatment with indomethacin.

The results of ex vivo platelet aggregation studies were also consistent with a role for platelet inhibitory prostaglandins in mediating the antiplatelet effects of U63,557a. Thus, inhibition of arachidonate-induced platelet aggregation by U63,557a was dependent on the concentration of arachidonic acid. At $0.08-0.17 \mathrm{mM}$ arachidonic acid, U63,557a failed to inhibit platelet aggregation, consistent with the proaggregatory effects of prostaglandin endoperoxides. At higher concentrations of arachidonic acid, platelet activation was inhibited in the presence of U63,557a. This was also demonstrated with a second TXA $_{2}$ synthase inhibitor, CGS13080, suggesting that it was a specific effect of this class of compounds. After incubation of platelets with a high concentration of arachidonic acid and U63,557a, platelets failed to respond to ADP or U46619. This effect was not seen at lower concentrations of arachidonic acid and was prevented by pretreating the platelets with aspirin.

These findings suggest that platelet inhibition in this setting is due to formation of a cyclooxygenase product. Similar findings have been reported using human platelets. Indeed, inhibition of arachidonate-induced aggregation of human platelets by $\mathrm{TXA}_{2}$ synthase inhibitors in vitro has been reported to largely reflect conversion of prostaglandin endoperoxides to $\mathrm{PGD}_{2}$ by the isomerase activity of plasma proteins $(24,25)$. However, unlike canine platelets, human platelets do not demonstrate increased inhibition at higher concentrations of arachidonic acid $(24,25)$. $\mathrm{PGD}_{2}$ inhibits canine platelets but only at concentrations one to two orders of magnitude greater than that required to inhibit human platelets (Fitzgerald, D. J., and G. A. FitzGerald, unpublished data). This may explain why inhibition of canine platelets is seen only at higher concentrations of arachidonic acid, where sufficient $\mathrm{PGD}_{2}$ may be formed to overwhelm the proaggregatory effect of prostaglandin endoperoxides. Whether this mechanism is operative in vivo in the dog, therefore, will depend on the amount of substrate released at the site of platelet activation.

In conclusion, these studies demonstrate that prostaglandin endoperoxides modulate the response to $\mathrm{TXA}_{2}$ synthase inhibition in a chronic, close-chest model of coronary thrombosis. First, endoperoxides may limit the response by substituting for the proaggregatory effect of $\mathrm{TXA}_{2}$. Second, they may be metabolized to platelet-inhibitory prostaglandins. The functional significance of the shunting of prostaglandin endoperoxides to platelet inhibitory products can be demonstrated when their proaggregatory effect is prevented by an antagonist of the shared $\mathrm{TXA}_{2}$ /prostaglandin endoperoxide receptor. The resulting synergistic interaction between a $\mathrm{TXA}_{2}$ synthase inhibitor and a $\mathrm{TXA}_{2}$ /prostaglandin endoperoxide receptor antagonist renders this combination a very effective antiplatelet regimen of potential therapeutic importance.

\section{Acknowledgments}

We gratefully acknowledge technical assistance of Patricia Price and gifts of U63,557a from Dr. Robert Gorman (Upjohn Company, Kalamazoo, MI), L636,499 from Dr. Anthony Ford-Hutchinson (MerckFrosst, Pointe Claire-Dorval, Quebec Canada) and CGS13080 from Dr. Frank Douglas (Ciba Geigy, Summit, NJ).

Supported by grants from the National Institutes of Health (HL-30400 and BRSG RR-05424-25-53). Dr. Desmond Fitzgerald is a recipient of a Faculty Development Award from the Pharmaceutical Manufacturers Association Foundation. Dr. Garret FitzGerald is an Established Investigator of the American Heart Association.

\section{References}

1. Granstrom, E., U. Diczfalusy, M. Hamberg, C. Malmsten, and B. Samuelsson. 1982. Thromboxane $\mathrm{A}_{2}$ : biosynthesis and effect on platelets. Adv. Prostaglandin Leuk. Res. 10:15-57.

2. Fitzgerald, D. J., L. Roy, F. Catella, and G. A. FitzGerald. 1986. Platelet activation in unstable coronary artery disease. $N$. Engl. J. Med. 315:983-989.

3. Lewis, H. D., J. W. Davis, D. G. Archibald, W. E. Steinke, T. C. Smitherman, J. E. Doherty, H. W. Schanper, M. M. LeWinter, E. Linares, J. M. Pouget, S. C. Sabharwarl, E. Chester, and H. DeMots. 1983. Protective effect of aspirin against acute myocardial infarction and death in men with unstable angina. N. Engl. J. Med. 309:396-403.

4. Cairns, J. A., M. Gent, J. Singer, K. J. Finnie, G. M. Froggat, D. A. Holder, G. Jablonsky, W. J. Kostuk, L. J. Melendez, M. G. G. Myers, D. L. Sackett, J. A. Sealy, and P. H. Tanser. 1985. Aspirin, sulphinpyrazone, or both in unstable angina. N. Engl. J. Med. 313:1369-1375.

5. The Steering Committee of the Physicians' Health Study Research Group. 1988. Preliminary report: findings from the aspirin component of the ongoing Physicians' Health Study. N. Engl. J. Med. 316:262-264.

6. Fitzgerald, D. J., F. Catella, L. Roy, and G. A. FitzGerald. 1988. 
Marked platelet activation in vivo after intravenous streptokinase. Circulation. 77:142-150.

7. FitzGerald, G. A., I. A. G. Reilly, and A. K. Pedersen. 1985. The biochemical pharmacology of thromboxane synthase inhibition in man. Circulation. 72:1194-1201.

8. Reilly, I. A. G., J. B. Doran, B. Smith, and G. A. FitzGerald. 1986. Increased thromboxane biosynthesis in a human preparation of platelet activation: biochemical and functional consequences of selective inhibition of thromboxane synthase. Circulation. 73:1300-1309.

9. FitzGerald, G. A., A. R. Brash, J. A. Oates, and A. K. Pederson. 1983. Endogenous prostacyclin biosynthesis and platelet function during selective inhibition of thromboxane synthase. J. Clin. Invest. 72:1336-1343.

10. Bunting, S. R., R. Gryglewski, A. S. Moncada, and J. R. Vane. 1976. Arterial walls generate from prostaglandin endoperoxides a substance (prostaglandin $\mathrm{X}$ ) which relaxes strips of mesenteric and coeliac arteries and inhibits platelet aggregation. Prostaglandins. 12:897-913.

11. Aiken, J. W., R. J. Shebuski, O. V. Miller, and R. R. Gorman. 1981. Endogenous prostacyclin contributes to the efficacy of a thromboxane synthase inhibitor for preventing coronary artery thrombosis. J. Pharmacol. Exp. Ther. 219:299-308.

12. Wu, K. K., Y. C. Chen, E. Fordham, C. H. Ts'Ao, G. Rayudu, and D. Matayoshi. 1981. Differential effects of two doses of aspirin on platelet-vessel wall interaction in vivo. J. Clin. Invest. 68:382-387.

13. FitzGerald, G. A., B. Smith, A. K. Pedersen, and A. R. Brash. 1984. Prostacyclin biosynthesis is increased in patients with severe atherosclerosis and platelet activation. N. Engl. J. Med. 310:10651068.

14. Reilly, I. A. G., and G. A. FitzGerald. 1986. Biosynthesis of thromboxane in patients with systemic sclerosis. Br. Med. J. 292:1037-1039.

15. Needleman, P., A. Raz, J. Ferrendelli, and M. Minkes. 1977. Application of imidazole as a selective inhibitor of thromboxane synthetase in human platelets. Proc. Natl. Acad. Sci. USA. 74:1716-1720.

16. Hepinstall, S. J., S. R. Bevan, S. P. Cockbill, and M. S. Parry. 1980. Effects of a selective inhibitor of thromboxane synthase on human blood platelet behaviour. Thromb. Res. 20:219-230.

17. Grimm, L. J., D. R. Knapp, D. Senator, and P. V. Halushka. 1981. Inhibition of platelet thromboxane synthesis by 7-(1-imadazolyl) heptanoic acid: dissociation from inhibition of aggregation. Thromb. Res. 24:304-317.

18. Randall, M. J., and R. I. R. Wilding. 1983. Acute arterial thrombosis in rabbits: reduced platelet accumulation after treatment with dazoxiben hydrochloride (UK-37,248-01). Br. J. Clin. Pharmacol. 15:49S-55S.

19. Hook, B. G., W. A. Schumacher, D. L. Lee, S. R. Jolly, and B. R. Lucchessi. 1985. Experimental coronary artery thrombosis in the absence of thromboxane $A_{2}$ synthesis: evidence for alternate pathways for coronary thrombosis. J. Cardiovasc. Pharmacol. 7:174-181.

20. Lewis, G. P., and J. R. Smith. 1984. Prostaglandin endoperoxides and thromboxane $A_{2}$ in thrombus formation in the hamster cheek pouch in vivo. Prostaglandins. 28:29-41.

21. Bush, L. R., W. B. Campbell, M. Buja, G. D. Tilton, and J. T. Willerson. 1984. Effects of the selective thromboxane synthetase inhibitor dazoxiben on variations in cyclical flow in stenosed canine coronary arteries. Circulation. 69:1161-1170.

22. Marcus, A. J., B. B. Weksler, E. A. Jaffe, and M. J. Broeckman. 1980. Synthesis of prostacyclin from platelet-derived endoperoxides by cultured endothelial cells. J. Clin. Invest. 66:979-986.

23. Schafer, A. I., D. D. Crawford, and M. S. Gimbrone. 1984. Unidirectional transfer of prostaglandin endoperoxides between platelets and endothelial cells. J. Clin. Invest. 73:1 105-1 108.

24. Patscheke, H. 1985. Thromboxane synthase inhibition potentiates washed platelet activation by endogenous and exogenous arachidonic acid. Biochem. Pharmacol. 34:1151-1156.

25. Bertele, V. A., A. Falanga, M. Tomasiak, C. Cerletti, and G. DeGaetano. 1984. SQ22436, an adenylate cyclase inhibitor, prevents the antiplatelet effects of dazoxiben, a thromboxane synthase inhibitor. Thromb. Haemostasis. 51:125-128.
26. Gresele, P., J. Arnout, H. Deckmyn, E. Huybrechts, G. Pieters, and J. Vermylen. 1987. Role of proaggregatory and antiaggregatory prostaglandins in hemostasis. Studies with combined thromboxane synthase inhibition and thromboxane receptor antagonism. J. Clin. Invest. 80:1435-1445.

27. Romson, J. L., D. W. Haack, and B. R. Luchessi. 1980. Electrical induction of coronary artery thrombosis in the ambulatory canine: a model for in vivo evaluation of antithrombotic agents. Thromb. Res. 17:841-853.

28. Fitzgerald, D. J., J. Doran, E. Jackson, and G. A. FitzGerald. 1986. Coronary vascular occlusion mediated via thromboxane $A_{2} /$ prostaglandin endoperoxide receptor activation in vivo. J. Clin. Invest. 77:496-502.

29. Roberts, L. J., B. J. Sweetman, and J. A. Oates. 1981. Metabolism of thromboxane $B_{2}$ in man. J. Biol. Chem. 256:8384-8391.

30. FitzGerald, G. A., A. R. Brash, P. Falardeau, and J. A. Oates. 1981. Estimated rate of prostacyclin secretion into the circulation in normal man. J. Clin. Invest. 68:1272-1276.

31. Born, G. V. R., and M. J. Gross. 1963. The aggregation of blood platelets. J. Physiol. (Lond.). 168:178-195.

32. Chignard, M., and B. B. Vargaftig. 1976. Dog platelets fail to aggregate when they form aggregating substances upon stimulation with arachidonic acid. Eur. J. Pharmacol. 38:7-18.

33. Lawson, J. A., A. R. Brash, J. Doran, and G. A. FitzGerald. 1985. Measurement of urinary 2,3-dinor-thromboxane $B_{2}$ and thromboxane $B_{2}$ using bonded-phase phenylboronic acid columns and capillary gas chromatography-negative ion chemical ionization mass spectrometry. Anal. Biochem. 150:463-470.

34. Nowak, J., J. J. Murray, J. A. Oates, and G. A. FitzGerald. 1987. Biochemical evidence of a chronic abnormality in platelet and vascular function in healthy individuals who smoke cigarettes. $\mathrm{Circu}$ lation. 76:6-14.

35. Fitzpatrick, F. A., R. R. Gorman, J. C. McGuire, R. C. Kelly, M. A. Wylanda, and F. F. Sun. 1977. A radioimmunoassay for thromboxane $\mathrm{B}_{2}$. Anal. Biochem. 83:1-7.

36. Siegel, S. 1956. Non-parametric statistics for the behavioural sciences. McGraw-Hill Book Co., Inc., New York. 184.

37. Fitzgerald, D. J., J. Fragetta, and G. A. FitzGerald. 1986. A chronic canine model of coronary thrombosis; minimization of artifactual increases in prostacyclin and thromboxane synthesis in vivo. Clin. Res. 34:298a. (Abstr.)

38. Fitzgerald, G. A., A. K. Pedersen, and C. Patrono. 1983. Analysis of thromboxane and prostacyclin biosynthesis in cardiovascular disease. Circulation. 67:1174-1175.

39. Chan, C. C., D. J. Nathaniel, P. J. Yusko, R. A. Hall, and A. W. Ford-Hutchinson. 1984. Inhibition of prostanoid-mediated platelet aggregation in vivo and in vitro by 3-hydroxymethyl-dibenzo (b,f) thiepin 5,5-dioxide (L-640,035). J. Pharmacol. Exp. Ther. 299:276282.

40. Carrier, R. E., E. J. Cragoe, E. Ethier, A. W. Ford-Hutchinson, Y. Girard, R. A. Hall, P. Hamel, J. Rokach, N. N. Share, C. A. Stone, and P. Yusko. 1984. Studies on L-640,035: a novel antagonist of contractile prostanoids in the lung. Br. J. Pharmacol. 82:389-396.

41. Hornby, E. J., and I. F. Skidmore. 1984. Reductions in thromboxane $\mathrm{B} 2$ production as a consequence of thromboxane receptor blockade in human platelet rich plasma. Br. J. Pharmacol. 83:432p. (Abstr.).

42. Menys, V. C., and J. A. Davies. 1984. Thromboxane-mediated activation of platelets and enhancement of platelet uptake onto collagen-coated glass or deendothelialized rabbit aorta. Lab. Invest. 50:184-189.

43. Gorman, R. R., R. A. Johnson, R. H. Spilman, and J. W. Aiken. 1983. Inhibition of platelet thromboxane $A_{2}$ synthase activity by sodium 5-(3'-pyridinylmethyl) benzofuran-2-carboxymethyl. Prostaglandins. 26:325-342.

44. Hamberg, M., J. Svensson, T. Wakabayashi, and B. Samuelsson. 1974. Isolation and structure of two prostaglandin endoperoxides that cause platelet aggregation. Proc. Natl. Acad. Sci. USA. 71:345349. 